\title{
Carotid sinus syndrome after carotid artery surgery
}

\author{
A. Dees, R.J. Batenburg de Jong ${ }^{1}$, C.A. Meeuwis ${ }^{1}$, M.F. de Boer ${ }^{1}$ \\ and P.W. de Leeuw ${ }^{2}$
}

Departments of Medical Oncology and ${ }^{1}$ Head and Neck Surgery, Rotterdam Cancer Institute, Rotterdam, and ${ }^{2}$ Department of Internal Medicine, University Hospital Maastricht, The Netherlands

\begin{abstract}
Summary: A 63 year old woman had been intensively treated for recurrent carcinoma of the neck. Following acute vascular surgery of the carotid artery, she developed the vasodilatory type of the carotid sinus syndrome. The presentation of this type of the syndrome was remarkable, since it is usually associated with primary or metastatic carcinoma in the neck region. Previous cancer treatment may have modified the course of disease in this patient, which ultimately had a lethal outcome.
\end{abstract}

\section{Introduction}

The carotid sinus syndrome (CSS) refers to dizziness or (near-) syncope resulting from a hyperactive carotid sinus reflex. The syndrome has been described in a number of benign and malignant diseases, involving the cervical region. ${ }^{1}$ Classically, the reflex has its origin in the carotid sinus, but branches of the glossopharyngeal or trigeminal nerves may be involved as well. In the face of increased carotid sinus stimulation, the vasomotor centre in the medulla reflexly causes withdrawal of sympathetic activity (hypotension) and/or enhancement of vagal tone (bradycardia). Clinical effects of the CSS are, therefore, classified as cardio-inhibitory, vasodilatory or mixed, depending on whether stimulation of the vagal nerve or inhibition of the sympathetic trunk predominates. In malignancy-associated CSS, often a mixed response is observed, but isolated vasodilatation, not responding to pacing therapy, has been described occasionally. ${ }^{2}$ In a number of cases CSS has been the presenting symptom of primary or metastatic disease and resolution of the syndrome has been observed after adequate antitumour treatment. ${ }^{3}$

We report here on a patient who was treated successfully in the past for recurrent carcinoma of the neck. However, during follow-up she developed the vasodilatory type of CSS after ligation of the common carotid artery.

Correspondence and present address: A. Dees, M.D., Department of Medicine, Ikazia Ziekenhuis, Montessoriweg 1, 3083 AN Rotterdam, The Netherlands. Accepted: 29 November 1991

\section{Case report}

A 63 year old female underwent emergency surgery for bleeding from a tear in the right common carotid artery. Seven years earlier she had been treated extensively by radical neck dissection, radiation treatment and chemotherapy for carcinoma of the piriform sinus. As the tissue appeared to be very fragile the artery could not be repaired and was, therefore, ligated. Postoperatively, the blood pressure was $130 / 70 \mathrm{mmHg}$ and heart rate was 72 beats $/ \mathrm{min}$. In the right supraclavicular fossa, a large ulcerative skin defect was found. Digital subtraction angiography confirmed occlusion of the right common carotid artery.

Two weeks later attacks of dizziness occurred, during which the blood pressure dropped to 70/ $40 \mathrm{mmHg}$, while the heart rate remained regular at 55 beats $/ \mathrm{min}$. She was unable to leave her bed and her blood pressure remained at a level of $90 /$ $60 \mathrm{mmHg}$. An electrocardiogram showed regular sinus rhythm with first-degree atrioventricular block only. Laboratory tests revealed a haemoglobin level of $10.7 \mathrm{~g} / \mathrm{dl}$, blood cell count, platelet count, electrolytes and renal function were normal. Cortisol was $0.46 \mu \mathrm{mol} / 1(n 0.20-0.70)$. Evaluation of catecholamines and pressor hormones at rest while blood pressure was $90 / 60 \mathrm{mmHg}$ revealed that noradrenaline was $1.11 \mathrm{nmol} / 1(n 0.5-3.0)$, adrenaline $0.10 \mathrm{nmol} / 1 \quad(n 0.10-1.0)$, dopamine $0.21 \mathrm{nmol} / 1 \quad(n 0.10-0.80), \quad$ renin $\quad 4.0 \mathrm{mU} / 1$ $(n 15-40)$, angiotensin II $6.6 \mathrm{pmol} / 1$ ( $n 25)$ and aldosterone $80 \mathrm{pmol} / 1(n 60-600)$. A few days later the patient developed fever, up to $40^{\circ} \mathrm{C}$, and her neck became warm and extremely painful at the right side. Marked oedema was observed. Computerized tomography, sonography, needle suction 
of the neck and cultures were negative for recurrent carcinoma or an abscess.

Tobramycin and penicillin were started and the temperature normalized. However, periods of severe hypotension persisted and despite treatment with atropine sulphate intravenously $(0.5 \mathrm{mg} 4$ times a day), heart rate remained unchanged. A diagnosis of CSS was made and arterial revascularization was contemplated but rejected on technical grounds. Six weeks after admission the patient died due to rupture of the subclavian artery.

\section{Discussion}

The patient described here had been treated with extensive surgery and radiation of the neck. Previously no signs of excitation or secondary hypersensitivity of the contralateral carotid sinus had been noted. She developed the carotid sinus syndrome following carotid artery ligation, which was complicated by oedema and high fever suggestive of local infection, although cultures remained negative. As radiation therapy has a destructive effect on baroreceptors, ${ }^{4}$ it is unlikely that mechanical compression of the carotid sinus played a role in the pathogenesis of the CSS. However, Cicogna et al. reported a patient who had been treated with radiation therapy of the neck, had fibrotic skin lesions without signs of tumour recurrence, and CSS, which proved to be unresponsive to cardiac pacing and carotid sinus denervation. ${ }^{2}$

\section{References}

1. Holmes, F.A., Glass, J.P., Ewer, M.S., Terjanian, T. \& Tetu, B. Syncope and hypotension due to carcinoma of the breast metastatic to the carotid sinus. Am J Med 1987, 82: $1238-1242$.

2. Cicogna, R., Curnis, A., Dei Cas, L. \& Visioli, O. Syncope and tumours in the neck: carotid sinus or glossopharyngeal syndrome? Eur Heart $J$ 1985, 6: 979-984.

3. Tulchinsky, M. \& Krasnow, S.H. Carotid sinus syndrome associated with an occult primary nasopharyngeal carcinoma. Arch Int Med 1988, 148: 1217-1219.

4. Greely, H.P., Smedal, M.I. \& Most, W. The treatment of the carotid sinus syndrome by irradiation. $N$ Engl J Med 1955, 252:
91-94.
In patients with benign lesions of the neck and CSS, syncope due to bradycardia is commonly observed, whereas pure vasodilatation-related syncope accounts for only $5 \%$ of cases. In malignancy-related CSS, pure vasodilatation occurs more frequently. The symptoms and signs in our patient are compatible with a diagnosis of the vasodilatory type of CSS. Although bradycardia was documented, there was no response to atropine suggesting that sympathetic withdrawal rather than enhanced vagal tone was responsible for the slowing of the pulse.

The laboratory data certainly are consistent with the theory that sympathetic withdrawal is a likely mechanism in the vasodilatory type of CSS. ${ }^{5}$ Evaluation of pressor hormones revealed reduced levels of renin and adrenaline, while noradrenaline and dopamine were normal. However, taking into account that blood pressure at the time of the sampling was fairly low, one would have expected higher levels of catecholamines in relation to the hypotensive stress. Dykman et al. have shown that under normal circumstances hypotensive stress results in an instantaneous increased in plasma noradrenaline. ${ }^{6}$ However, during a hypotensive attack due to CSS, noradrenaline remains low.

In conclusion, the case presented here demonstrates a vasodilatory type of the CSS following 0 carotid artery surgery for carcinoma of the neck. Although not proven, it cannot be excluded that the occurrence of this type of CSS was determined, to some extent, by the previous treatment.

5. Onrot, J., Wiley, R.G., Fogo, A., Biaggioni, I., Robertson, D. \& Hollister, A.S. Neck tumour with syncope due to paroxysmal sympathetic withdrawal. J Neurol Neurosurg Psychiatry 1987, 50: 1063-1066.

6. Dykman, I.R., Montgomery, E.B., Gerstenberg, P.D., Zieger, E.H., Chutter, W.E. \& Cryer, P.E. Glossopharyngeal neuralgia with syncope secondary to tumour. Treatment and pathophysiology. Am J Med 1981, 71: 165-168. 